LÚCIO AD; SANTOS D; CARGNELUTTI FILHO A; SCHABARUM DE. 2016. Método de Papadakis e tamanho de parcela em experimentos com a cultura da alface. Horticultura Brasileira 34: 066-073. DOI - http://dx.doi.org/10.1590/S0102-053620160000100010

\title{
Método de Papadakis e tamanho de parcela em experimentos com a cultura da alface
}

\author{
Alessandro D Lúcio; Daniel Santos; Alberto Cargnelutti Filho; Denison E Schabarum \\ Universidade Federal de Santa Maria (UFSM), Santa Maria-RS, Brasil; adlucio@ufsm.br; danielsantosagro@gmail.com; alberto. \\ cargnelutti.filho@gmail.com; denisonesequiel@yahoo.com.br
}

\section{RESUMO}

O trabalho teve por objetivo testar a eficiência do método Papadakis na melhoria da qualidade de experimentos com a cultura da alface, determinar a melhor forma de estimativa da covariável e o tamanho ótimo de parcela para uso no método. A forma de estimativa da covariável que proporciona maior eficiência do método e o cálculo do tamanho de parcela ajustado para uso nesse método, foram determinados a partir de oito ensaios de uniformidade com a cultura da alface. A eficácia do uso de análise de covariância (ANCOVA) utilizando a covariável estimada pelo método Papadakis foi testada a partir de um experimento com tratamentos com a cultura da alface, em que a variável estudada foi a massa fresca da parte aérea das plantas. O uso de ANCOVA com a covariável estimada pelo método Papadakis melhora a qualidade de experimentos com a cultura da alface. A covariável que proporciona a maior eficiência da ANCOVA é aquela que considera uma parcela vizinha de cada lado da parcela de referência no sentido da linha de cultivo. O tamanho de parcela na linha de cultivo, ajustado para uso do método, é de quatro plantas $(1,2 \mathrm{~m})$ para estufa e túnel, e de cinco plantas $(1,5 \mathrm{~m})$ para o campo.

Palavras-chave: Lactuca sativa, análise de covariância, precisão experimental, coeficiente de variação.

\begin{abstract}
Papadakis method and plot size in experiments with lettuce

This study aimed to evaluate the efficiency of Papadakis method in improving quality of the trials in the lettuce crop, establish the best way to estimate the covariate and the optimal plot size to be used in this method. The way of estimating the covariate that allows a better efficiency of the method and the estimation of the plot size adjusted to be used in this method were determined from eight uniformity essays on the lettuce crop. The efficacy of using the analysis of covariance (ANCOVA), using a covariate estimated by the Papadakis method, was tested in an experiment with treatments on the lettuce crop, the variable studied being the aboveground part fresh mass of the plants. The use of ANCOVA with the covariate projected by the Papadakis method improves the quality of the experiments within the lettuce crop. The covariate that provides the best efficiency of ANCOVA is the one that considers a close share of each side of the relative share in the cropping line. The plot size on the cropping line, adjusted to be used in the method, is of four plants $(1.2 \mathrm{~m})$ for greenhouse and tunnel and five plants $(1.5 \mathrm{~m})$ for the field.
\end{abstract}

Keywords: Lactuca sativa, covariance analysis, experimental accuracy, coefficient of variation.

\section{(Recebido para publicação em 5 de junho de 2014; aceito em 7 de agosto de 2015) (Received on June 5, 2014; accepted on August 7, 2015)}

\begin{abstract}
A alface é a hortaliça folhosa mais produzida no Brasil (ABCSEM, 2011), sendo seu cultivo, uma importante fonte de renda especialmente em pequenas propriedades rurais. Frente a isso, diversas pesquisas estão sendo realizadas com essa cultura visando encontrar formas de aumentar sua qualidade e produtividade (Radin et al., 2004; Oliveira et al., 2006; Baumgartner et al., 2007; Pimentel et al., 2009). Essas pesquisas são realizadas com o uso de experimentos, que devem ser planejados e conduzidos de modo que o erro experimental seja o mínimo possível, fornecendo assim aos usuários informações acuradas dos resultados de pesquisas.
\end{abstract}

Algumas particularidades inerentes às culturas olerícolas, tais como ponto de colheita subjetivo e manejo cultural mais intensivo em relação às outras culturas são fontes adicionais de variabilidade e consequentemente de erro experimental (Lorentz et al., 2005; Lúcio et al., 2008). Dessa forma, muitas vezes os experimentos com essas culturas não apresentam o erro experimental satisfatoriamente baixo.

Visando apresentar estratégias para minimizar o erro experimental com a cultura da alface, encontram-se na literatura alguns estudos nos quais foi buscado via a identificação do delineamento experimental e dos tamanhos de parcela e de amostra adequados (Marodim et al., 2000; Santos et al., 2010; Lúcio et al., 2011). No entanto, em alguns casos, mesmo após estas estratégias, o erro experimental ainda não se torna satisfatoriamente baixo, indicando a necessidade de novas abordagens estatísticas experimentais.

A análise de covariância é uma técnica consagrada para a melhoria da qualidade de experimentos (Storck et $a l ., 2006)$. Nessa técnica uma variável secundária (covariável) é mensurada concomitantemente à variável de interesse, para ajustar o erro experimental. Papadakis (1937) propôs um método de ajuste da variabilidade espacial em que a covariável para uso na análise de covariância, é obtida dos erros 
experimentais calculados entre as parcelas vizinhas. Nesse método existe a vantagem de não ser necessário mensurar a covariável concomitantemente à variável, uma vez que a mesma pode ser calculada somente no momento da análise. O método mostrou-se eficaz na redução do erro experimental em experimentos para culturas como feijoeiro, soja, milho, eucalipto (Cargnelutti Filho et al., 2003; Souza et al., 2003; Costa et al., 2005; Storck et al., 2008, 2009, 2010). Como a covariável é obtida a partir dos erros, não sendo influenciada pelo efeito dos tratamentos, o método Papadakis poderia ser usado também em ensaios em branco. Nessa situação seria possível estimar um tamanho de parcela a partir de dados ajustados pelo método Papadakis.

O tamanho de parcela depende principalmente da variabilidade da área experimental (Storck et al., 2006). Quando se faz uso da análise de covariância com a covariável estimada pelo método Papadakis, a variabilidade que estará agindo na área experimental será alterada. Isso torna importante que seja obtido um tamanho de parcela prevendo essa alteração na variabilidade.

São encontrados na literatura poucos trabalhos avaliando a eficiência ou indicando a forma adequada para aplicação do método Papadakis para cultura da alface. Assim, os objetivos deste estudo foram testar a eficiência do método Papadakis no aumento da qualidade em experimentos com alface, determinar a forma de obter a estimativa da covariável e estimar o tamanho de parcela para uso em experimentos em que se aplicará o método.

\section{MATERIAL E MÉTODOS}

O estudo foi realizado a partir de experimentos em branco oriundos do banco de dados do Setor de Experimentação Vegetal do Departamento de Fitotecnia da UFSM. Esses experimentos foram realizados na área experimental do Departamento de Fitotecnia, Campus da Universidade Federal de Santa Maria (UFSM), município de Santa Maria-RS

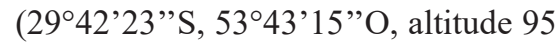
metros). O clima da região, segundo a classificação de Köeppen (Moreno, 1961) é do tipo Cfa, temperado chuvoso, com chuvas bem distribuídas ao longo do ano e subtropical do ponto de vista térmico. O solo é classificado no Sistema Brasileiro de Classificação de Solos (Embrapa, 2006) como Argissolo Vermelho Distrófico arênico.

Os experimentos utilizados no estudo constaram das condições de cultivo: 1) em estufa no verão; 2) a campo no verão; 3) em estufa no inverno; 4) a campo no inverno; 5) em estufa no outono; 6) em túneis no outono; 7) em estufa na primavera e; 8) em túneis na primavera. Em todos os experimentos foi utilizada foi a cultivar Vera e as plantas foram dispostas em camalhões (linhas de cultivo) espaçados de 1,0 m sem o uso de mulching, com espaçamento entre plantas de $0,3 \mathrm{~m}$. Nos experimentos realizados em estufa plástica foram utilizadas seis linhas de cultivo cada uma contendo 48 plantas. Nos experimentos realizados em túneis foram usadas três linhas de cultivo cada uma com 60 plantas. Nos experimentos realizados a campo, no verão foram utilizadas seis linhas e no inverno três linhas, cada uma contendo 48 plantas. A variável analisada nesses experimentos foi a massa fresca da parte aérea das plantas. $\mathrm{O}$ experimento em que utilizaram-se tratamentos, foi realizado a campo, utilizando 3 linhas de cultivo espaçadas de $1 \mathrm{~m}$, com 64 plantas espaçadas de $0,3 \mathrm{~m}$. O delineamento experimental foi de blocos ao acaso sendo a linha de cultivo o bloco. A parcela foi constituída por quatro plantas no sentido da linha de cultivo. Os tratamentos utilizados nesse experimento foram obtidos da combinação de diferentes doses de Acres-

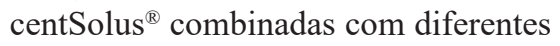
momentos de aplicação, resultando em 16 tratamentos (Schwertner, 2012).

A estufa utilizada possui estrutura metálica do tipo arco pampeano, pé direito de 2,0 $\mathrm{m}$ e 3,5 $\mathrm{m}$ na parte central, com $20 \mathrm{~m}$ de comprimento e $10 \mathrm{~m}$ de largura, orientada no sentido norte-sul. $\mathrm{O}$ túnel utilizado possui pé direito de $3,0 \mathrm{~m}, 20 \mathrm{~m}$ de comprimento e orientação norte-sul. A cobertura da estufa e do túnel foi realizada com filme de polietileno de baixa densidade (PEBD), com espessura de 150 micras e aditivo
anti-UV.

Para cada um dos experimentos em branco, foram ajustados os valores de cada parcela através da adaptação do método de Papadakis (Papadakis, 1937). Esse procedimento foi realizado em cada linha de cultivo considerando parcelas com 1, 2, 3, 4, 6 e 8 plantas, nos experimentos em estufa e a campo, e parcelas com 1, 2, 3, 4, 6 e 10 plantas nos experimentos em túnel.

Para fins de uso das equações, consideraram-se i parcelas e $\mathrm{j}$ linhas de cultivo. Inicialmente foi estimado o resíduo de cada parcela pela equação:

$$
R_{(i, j)}=Y_{(i, j)}-\bar{Y}_{(i, j)}
$$

em que, $R_{(i, j)}$ é o resíduo da parcela $\mathrm{i}$ na linha de cultivo $j ; Y_{(i, j)}$ é o valor da parcela i na linha de cultivo $\mathrm{j} ; \overline{\mathrm{Y}}_{(\mathrm{i}, \mathrm{j})}$ é a média das parcelas na linha de cultivo $\mathrm{j}$.

Em seguida foram estimados os resíduos médios de três formas de modo a determinar a abrangência na linha que possibilitará maior eficiência da covariável:

$C_{1 j}=\frac{\left[R_{(i, j)}+R_{(i-1, j)}+R_{(i+1, j)}\right]}{3}$ (2)
$C_{2 j}=\frac{\left[R_{(i, j)}+R_{(i-1, j)}+R_{(i-2, j)}+R_{(i+1, j)}+R_{(i+2, j)}\right]}{5}$

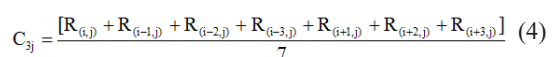

Quando um determinado resíduo estava na extremidade da linha de cultivo, ou próximo a ela, de modo que algum dos resíduos vizinhos não estava disponível, a covariável foi estimada através dos resíduos vizinhos disponíveis, adequando-se o denominador da equação ao número de resíduos disponíveis.

O ajuste do valor original de cada parcela usando a covariável foi realizado por meio da equação:

$$
\mathrm{Y}_{(\mathrm{i}, \mathrm{j})}^{*}=\mathrm{Y}_{(\mathrm{i}, \mathrm{j})}-\beta\left(\mathrm{C}_{(\mathrm{i}, \mathrm{j})}-\overline{\mathrm{C}}_{(\mathrm{.j})}\right)
$$

em que, $\mathrm{Y}_{(\mathrm{i}, \mathrm{j})}^{*}$ é a variável corrigida na parcela $\mathrm{i}$ da linha de cultivo $\mathrm{j} ; \mathrm{Y}_{(\mathrm{i}, \mathrm{j})}$ é a variável original na parcela i da linha de cultivo j; ß é o coeficiente de regressão entre a variável original e a covariável; $\mathrm{C}_{(\mathrm{i}, \mathrm{j})}$ é a covariável na parcela i da linha de cultivo j; $\overline{\mathrm{C}}_{(\cdot, \mathrm{j})}$ é a média da covariável na linha de cultivo j. Para verificar qual forma de estimativa da covariável que possibilitou maior eficiência ao método, calculou-se a variância e o coeficiente de variação para os dados ajustados e 
não ajustados.

Ainda com os dados dos experimentos em branco, para os dados ajustados e não ajustados, estimou-se o tamanho ótimo de parcela pelo método da curvatura máxima do coeficiente de variação proposto por Paranaíba et al. (2009):

$$
\mathrm{X}_{0}=\frac{10 \sqrt[3]{2\left(1-\hat{\rho}^{2}\right) \mathrm{S}^{2} \overline{\mathrm{Y}}}}{\overline{\mathrm{Y}}}
$$

em que, $\hat{X}_{0}$ é o tamanho ótimo de parcela; $\mathrm{S}^{2}$ é a variância na linha de cultivo; $\bar{Y}$ é a média das plantas na linha de cultivo; $\hat{\rho}$ é a autocorrelação espacial de primeira ordem, estimado pela equação . O coeficiente de variação do tamanho ótimo da parcela foi calculado pela equação

$$
\hat{\rho}=\frac{\sum_{i=2}^{n}\left(R_{(i, j)}\right)\left(R_{(i-1, j)}\right)}{\sum_{i=1}^{n}\left(R_{(i, j)}\right)^{2}}
$$

O coeficiente de variação do tamanho ótimo da parcela foi calculado pela equação

$$
\mathrm{CV}_{\left(\mathrm{X}_{0}\right)}=\frac{100 \sqrt{\left(1-\hat{\rho}^{2}\right)^{\mathrm{S}^{2} / \bar{Y}^{2}}}}{\sqrt{\mathrm{X}_{0}}}
$$

Para os dados do experimento com tratamentos, realizou-se a análise de variância (ANOVA) e também a análise de covariância (ANCOVA), com a covariável estimada das três formas descritas anteriormente $\left(\mathrm{C}_{1 \mathrm{j}}, \mathrm{C}_{2 \mathrm{j}}\right.$ e $\left.\mathrm{C}_{3 \mathrm{j}}\right)$, calculadas considerando i tratamentos e $\mathrm{j}$ blocos. Nesse caso os resíduos utilizados para cálculo das covariáveis foram obtidos subtraindo o valor da parcela pela média do respectivo tratamento.

A avaliação da precisão experimental, quando utilizado ANOVA e ANCOVA, foi procedida com base na estimativa das estatísticas: Coeficiente de variação residual (CV\%); F calculado para tratamento $\left(\mathrm{F}_{\text {calculado }}\right)$; Quadrado médio do erro $\left(\mathrm{QM}_{\mathrm{E}}\right)$; nível mínimo de significância associado ao teste $\mathrm{F}$ para efeito de tratamento (P-valor); diferença mínima significativa entre dois tratamentos pelo teste de Tukey (DMS):

(DMs): $\Delta=q_{\alpha\left(n ; G L_{E}\right)} \sqrt{\frac{Q M_{E}}{J}}(9)$, em que, $q_{\alpha\left(n ; G L_{E}\right)}$ é o valor da tabela do teste de Tukey, n é o número de tratamentos, $\mathrm{GL}_{\mathrm{E}}$ é número de graus de liberdade do erro, $\mathrm{QM}_{\mathrm{E}}$ é o quadrado médio do erro, J é o número de repetições; diferença mínima significativa pelo teste de Tukey em porcentagem da média (DMS\%):

$$
D M S=\frac{100 * \Delta}{\hat{m}}
$$

em que, $\hat{m}$ é a estimativa da média geral do ensaio.

Antes da realização da ANOVA e da ANCOVA foram testados os pressupostos: aditividade dos efeitos do modelo, normalidade, homogeneidade e aleatoriedade dos erros. Além disso, para realização da ANCOVA, testaram-se os seguintes pressupostos: a covariável é independente do efeito de tratamentos; o coeficiente de regressão da variável sobre a covariável, depois de removidos os efeitos de tratamentos e de blocos, é linear independente dos tratamentos e blocos. A pressuposição de que a covariável é independente do efeito de tratamento foi testada através de uma análise de variância para a covariável. As análises estatísticas foram realizadas no software SOC/NTIA/Embrapa e no aplicativo Office Excel. Todas as análises foram realizadas admitindo-se 5\% de probabilidade de erro.

\section{RESULTADOS E DISCUSSÃO}

Houve atendimento aos pressupostos da aditividade dos efeitos do modelo, normalidade, homogeneidade e aleatoriedade dos erros, mostrando que é correto realizar a análise dos dados por estatística paramétrica. Além disso, a covariável calculada foi independente do efeito de tratamentos e o coeficiente de regressão da variável sobre a covariável foi linearmente independente dos tratamentos e blocos. Isso demonstra que, se a covariável for eficiente para o ajuste da covariável, é correto o uso da análise de covariância utilizando as covariáveis testadas.

Em 100\% das situações testadas (ambientes, tamanhos de parcela e estações de cultivo), o ajuste com a covariável estimada pelo método Papadakis gerou redução do coeficiente de variação (CV\%), indicando redução na variabilidade (Tabelas 1 e 2). A partir desses resultados é possível inferir que o uso da covariável calculada pelo método será eficiente na redução do erro experimental em experimentos com a cultura da alface em que se testam tratamentos, tal qual ocorre em experimentos com outras culturas (Cargnelutti Filho et al., 2003; Souza et al., 2003; Costa et al., 2005; Storck et al., 2008, 2009, 2010).

Dentre as formas de ajuste, a melhor foi aquela realizada pela covariável $\mathrm{C} 1$. Essa forma possibilitou o menor valor do coeficiente de variação $(\mathrm{CV})$ em relação ao não ajustado, em 95,8, 94,4 e $100 \%$ dos testes, respectivamente, na estufa, túnel e campo. A forma de estimativa da covariável que possibilitou o pior resultado em termos de redução de $\mathrm{CV}$ foi a $\mathrm{C} 3$, onde ocorreu a menor redução do $\mathrm{CV}$ em 75,0, 88,9 e 83,3\% dos testes, respectivamente na estufa, túnel e campo (Tabelas 1 e 2). Resultados similares foram obtidos por Cargnelutti Filho et al. (2003) para milho, no qual a melhor forma de cálculo da covariável foi a que considerou um vizinho de cada lado da parcela de referência. Dessa forma a covariável C1 deve ser preferida para uso na ANCOVA em experimentos com alface, pois na maioria das situações possibilitará um melhor resultado em termos de melhoria da qualidade do experimento. A covariável C3 deve ser evitada, pois reduzirá o potencial de melhoria de qualidade do experimento com o uso da ANCOVA com covariável calculada pelo método Papadakis. Um dos motivos para a covariável C3 ter sido a menos eficiente, pode ser a elevada variabilidade existente nos ambientes protegidos cultivados com hortaliças (Lorentz et al., 2005; Lúcio et al., 2008), fazendo com que vizinhos mais distantes da parcela de referência tenham menos a informar sobre esta parcela do que os vizinhos mais próximos.

Para todas as situações em que houve o ajuste pela covariável obtida pelo método Papadakis, houve a redução no tamanho ótimo da parcela. O menor tamanho de parcela ocorreu para o ajuste com a covariável C1 (Tabela 3). A possibilidade de usar menores tamanhos de parcela para o ajuste com a covariável $\mathrm{C} 1$ ocorre, pois o tamanho de parcela está diretamente relacionado à variabilidade existente, e essa foi menor 
no ajuste pela $\mathrm{C} 1$. Além da redução nos tamanhos de parcela, em todas as situações, os CV dos tamanhos de parcela foram menores para as situações em que houve o ajuste pelo método Papadakis (Tabela 3). Isso torna evidente os benefícios do uso do método, pois além de possibilitar a redução do tamanho de parcela, permite que os tamanhos de parcela possibilitem maior precisão aos experimentos.

Considerando um tamanho único de parcela por ambiente, para o ajuste pela covariável C1, o tamanho de parcela foi de quatro plantas para estufa e túnel, e de cinco plantas para o campo, o que equivale a uma abrangência na linha de cultivo de 1,2 m e 1,5 m, respectivamente. Em termos de número de plantas, houve redução no tamanho de parcela em relação ao não ajustado de $33 \%$

Tabela 1. Variância $\left(\mathrm{s}^{2}\right)\left(\mathrm{em} \mathrm{g} 10^{3}\right)$ e coeficiente de variação $\{\mathrm{CV}(\%)\}$ entre parcelas para massa fresca de alface cultivada em estufa e a campo de 2007, considerando diferentes tamanhos de parcela, sem ajuste (SA) e com ajuste através das covariáveis estimadas pelo método Papadakis $(\mathrm{C} 1, \mathrm{C} 2, \mathrm{C} 3)^{*}$ \{variance $\left(\mathrm{s}^{2}\right)\left(\right.$ in $\left.\mathrm{g} 10^{3}\right)$ and coefficient of variation $\{\mathrm{CV}(\%)\}$ among plots for fresh mass of lettuce grown under greenhouse and in the field in 2007, considering different plot sizes without adjustment (SA) and adjustment through the covariates estimated by Papadakis method (C1, C2, C3)*\}. Santa Maria, UFSM, 2013.

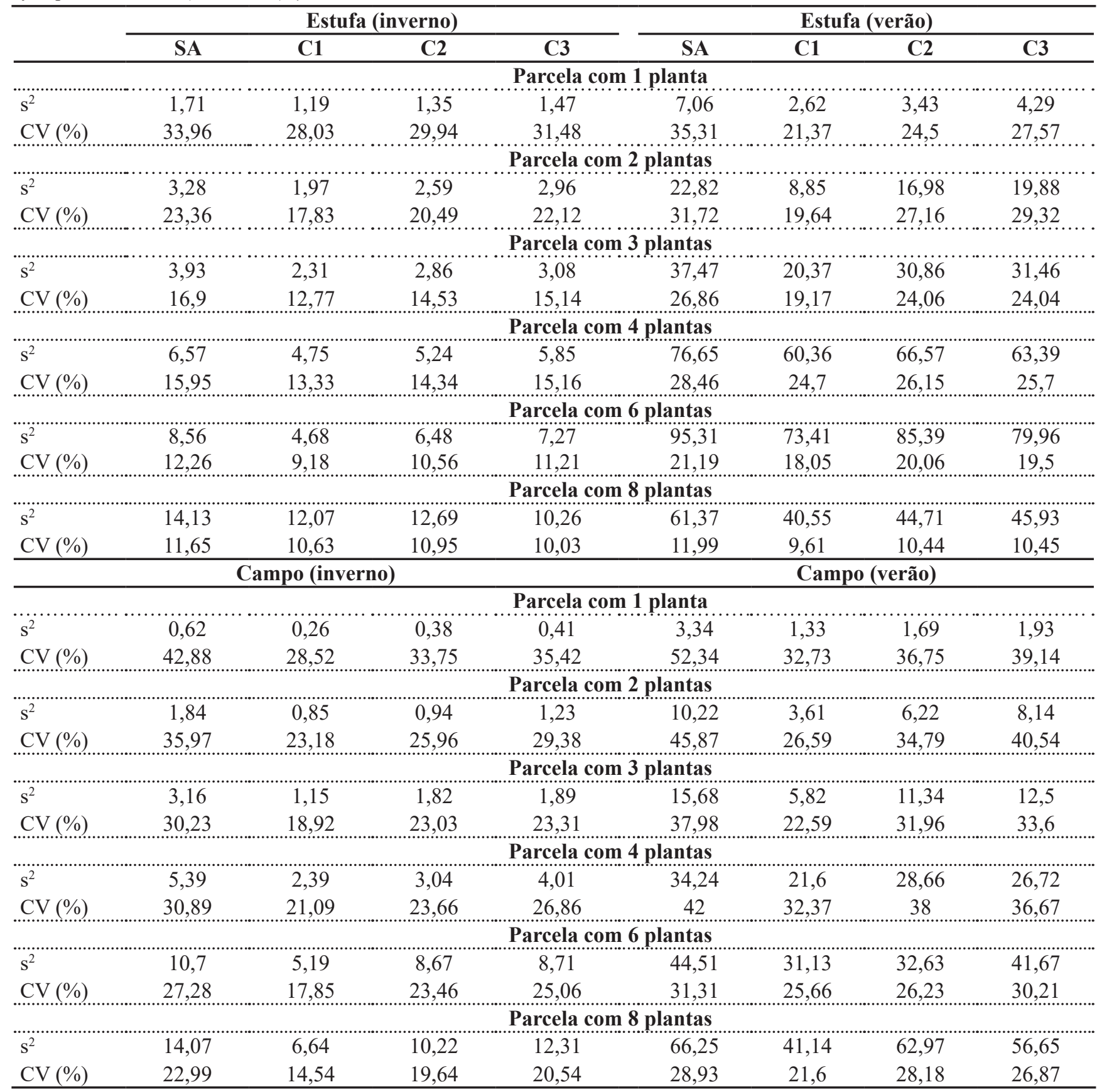

$\mathrm{C} 1=$ ajuste com a covariável estimada pela equação 2; $\mathrm{C} 2=$ ajuste com a covariável estimada pela equação 3; C3= ajuste com a covariável estimada pela equação 4 . 
Tabela 2. Variância $\left(\mathrm{s}^{2}\right)\left(\mathrm{em} \mathrm{g} 10^{3}\right)$ e coeficiente de variação $\{\mathrm{CV}(\%)\}$ entre parcelas para massa fresca de alface cultivada em estufa e túnel no ano de 2008, considerando diferentes tamanhos de parcela, sem ajuste (SA) e com ajuste através das covariáveis estimadas pelo método Papadakis $(\mathrm{C} 1, \mathrm{C} 2, \mathrm{C} 3)^{*}\left\{\right.$ variance $\left(\mathrm{s}^{2}\right)\left(\right.$ in $\left.\mathrm{g} 10^{3}\right)$ and coefficient of variation $\{\mathrm{CV}(\%)\}$ between plots for fresh mass of lettuce grown under greenhouse and tunnel, in 2008, considering different plot sizes without adjustment (SA) and adjustment through the covariates estimated by Papadakis method (C1, C2, C3)*\}. Santa Maria, UFSM, 2013.

\begin{tabular}{|c|c|c|c|c|c|c|c|c|c|c|c|c|}
\hline & \multicolumn{4}{|c|}{ Estufa (outono) } & \multicolumn{4}{|c|}{ Estufa (primavera) } & \multicolumn{4}{|c|}{ Túnel 1 (outono) } \\
\hline & SA & C1 & C2 & C3 & SA & C1 & C2 & C3 & SA & C1 & $\mathrm{C} 2$ & C3 \\
\hline & \multicolumn{12}{|c|}{ Parcela com 1 planta } \\
\hline $\mathrm{s}^{2}$ & 7,58 & 4,62 & 5,26 & 5,72 & 15,11 & 8,15 & 9,21 & 9,90 & 10,36 & 7,00 & 7,52 & 8,00 \\
\hline \multirow[t]{2}{*}{$\mathrm{CV}(\%)$} & 34,95 & 25,77 & 28,16 & 29,38 & 30,53 & 21,59 & 22,87 & 23,78 & 45,70 & 36,45 & 38,94 & 39,65 \\
\hline & \multicolumn{12}{|c|}{ Parcela com 2 plantas } \\
\hline $\mathrm{s}^{2}$ & 16,28 & 6,82 & 10,29 & 11,07 & 37,89 & 14,21 & 19,46 & 19,82 & 20,99 & 7,42 & 11,67 & 14,96 \\
\hline \multirow[t]{2}{*}{$\mathrm{CV}(\%)$} & 25,92 & 15,53 & 19,70 & 20,76 & 24,50 & 14,00 & 16,35 & 16,74 & 32,91 & 20,41 & 24,76 & 28,41 \\
\hline & \multicolumn{12}{|c|}{ Parcela com 3 plantas } \\
\hline $\mathrm{s}^{2}$ & 28,00 & 13,87 & 18,48 & 17,20 & 66,42 & 23,69 & 27,77 & 36,15 & 36,21 & 14,70 & 23,89 & 31,44 \\
\hline \multirow[t]{2}{*}{$\mathrm{CV}(\%)$} & 23,27 & 15,52 & 18,25 & 17,76 & 21,74 & 12,17 & 13,47 & 15,18 & 28,77 & 18,20 & 23,77 & 26,83 \\
\hline & \multicolumn{12}{|c|}{ Parcela com 4 plantas } \\
\hline $\mathrm{s}^{2}$ & 43,95 & 23,90 & 24,93 & 26,57 & 112,41 & 33,62 & 55,48 & 71,38 & 49,84 & 28,15 & 42,51 & 47,21 \\
\hline \multirow{2}{*}{$\mathrm{CV}(\%)$} & 21,84 & 15,73 & 16,17 & 17,00 & 21,28 & 11,02 & 14,10 & 15,99 & 25,00 & 18,66 & 23,12 & 24,27 \\
\hline & \multicolumn{12}{|c|}{ Parcela com 6 plantas } \\
\hline $\mathrm{s}^{2}$ & 71,45 & 24,23 & 44,98 & 51,63 & 210,20 & 61,59 & 132,26 & 158,27 & 67,98 & 51,32 & 67,20 & 66,62 \\
\hline \multirow[t]{2}{*}{$\mathrm{CV}(\%)$} & 18,77 & 10,92 & 15,03 & 16,04 & 19,34 & 9,88 & 13,97 & 15,19 & 20,53 & 18,02 & 20,38 & 20,34 \\
\hline & \multicolumn{8}{|c|}{ Parcela com 8 plantas } & \multicolumn{4}{|c|}{ Parcela com 10 plantas } \\
\hline $\mathrm{s}^{2}$ & 99,89 & 35,56 & 69,40 & 62,69 & 348,20 & 133,85 & 226,02 & 224,62 & 86,35 & 85,30 & 65,67 & 62,14 \\
\hline \multirow[t]{3}{*}{$\mathrm{CV}(\%)$} & 16,09 & 9,44 & 13,25 & 13,35 & 18,55 & 10,35 & 13,17 & 13,69 & 13,87 & 13,74 & 12,19 & 11,41 \\
\hline & \multicolumn{4}{|c|}{ Túnel 1 (primavera) } & \multicolumn{4}{|c|}{ Túnel 2 (outono) } & \multicolumn{4}{|c|}{ Túnel 2 (primavera) } \\
\hline & \multicolumn{12}{|c|}{ Parcela com 1 planta } \\
\hline $\mathrm{s}^{2}$ & 49,41 & 15,95 & 17,90 & 19,27 & 12,69 & 6,37 & 7,53 & 8,90 & 26,90 & 18,02 & 22,53 & 22,72 \\
\hline \multirow[t]{2}{*}{$\mathrm{CV}(\%)$} & 41,58 & 23,60 & 24,83 & 25,79 & 37,59 & 25,98 & 28,75 & $31,01 \ldots$ & 23,84 & 19,29 & 21,80 & 21,85 \\
\hline & \multicolumn{12}{|c|}{ Parcela com 2 plantas } \\
\hline $\mathrm{s}^{2}$ & 158,22 & 35,94 & 39,24 & 44,90 & 32,12 & 13,75 & 19,22 & 19,35 & 60,89 & 46,59 & 39,99 & 46,98 \\
\hline \multirow[t]{2}{*}{$\mathrm{CV}(\%)$} & 37,16 & 17,35 & 18,49 & 19,75 & 29,94 & 19,04 & 22,86 & 23,29 & 17,98 & 15,52 & 14,34 & 15,59 \\
\hline & & & & & Par & cela con & 3 plant & & & & & \\
\hline $\mathrm{s}^{2}$ & 329,69 & 55,82 & 80,27 & 92,38 & 61,25 & 31,43 & 34,62 & 37,27 & 73,70 & 30,70 & 45,71 & 44,26 \\
\hline $\mathrm{CV}(\%)$ & 35,73 & 14,62 & 17,58 & 18,82 & 27,48 & 19,69 & 20,69 & 21,65 & 13,05 & 7,94 & 9,96 & 10,08 \\
\hline & & & & & Par & ela con & 4 plant & & & & & \\
\hline $\mathrm{s}^{2}$ & 528,51 & 55,95 & 102,81 & 148,62 & 91,74 & 39,12 & 46,42 & 48,92 & 112,23 & 48,57 & 66,64 & 76,63 \\
\hline $\mathrm{CV}(\%)$ & 33,75 & 10,84 & 14,61 & 17,77 & 25,23 & 16,50 & 18,07 & 18,54 & 12,22 & 7,65 & 9,33 & 10,09 \\
\hline & & & & & Par & ela con & 6 plant & & & & & \\
\hline $\mathrm{s}^{2}$ & 1214,44 & 235,32 & 345,34 & 467,95 & 161,31 & 61,58 & 67,58 & 91,73 & 207,59 & 89,61 & 148,64 & 146,28 \\
\hline $\mathrm{CV}(\%)$ & 34,20 & 14,89 & 18,13 & 21,33 & 22,14 & 13,63 & 14,15 & 16,71 & 11,04 & 7,13 & 9,37 & 9,15 \\
\hline & & & & & Parc & ela com & 10 plan & & & & & \\
\hline $\mathrm{s}^{2}$ & 3153,05 & 664,26 & 1133,21 & 936,90 & 317,67 & 63,08 & 235,67 & 117,83 & 473,62 & 263,26 & 419,77 & 416,08 \\
\hline $\mathrm{CV}(\%)$ & 32,78 & 14,83 & 19,78 & 17,71 & 18,77 & 7,98 & 15,95 & 11,07 & 9,81 & 7,05 & 9,12 & 9,21 \\
\hline
\end{tabular}

$\mathrm{C} 1=$ ajuste com a covariável estimada pela equação 2 ; $\mathrm{C} 2=$ ajuste com a covariável estimada pela equação 3; C3= ajuste com a covariável estimada pela equação 4.

na estufa e túnel e $36 \%$ para o campo (Tabela 3). A necessidade de maiores tamanhos de parcela nos experimentos em campo corrobora os resultados obtidos por Lúcio et al. (2011), também para a cultura da alface.

Esses mesmos autores observaram que o uso de um tamanho de parcela inferior ao ótimo da cultura da alface, levaria a um aumento de até $60 \%$ da diferença mínima significativa entre médias em algumas situações. No entanto, muitas vezes, o pesquisador necessita usar tamanhos de parcela menores de modo a aumentar o número de repetições ou adequar o número de tratamentos à área experimental disponível. Esse problema pode ser contornado com o uso do tamanho de parcela ajustado para posterior uso da ANCOVA com a covariável estimada pelo método 
Tabela 3. Tamanho ótimo de parcela $\left(\mathrm{X}_{0}\right)$, em número de plantas, e coeficiente de variação entre parcelas do $\mathrm{X}_{0}\left(\mathrm{CV}_{(\mathrm{X} 0)}\right)$, em \%, para a massa fresca de alface em experimentos em estufa, túnel e a campo, sem ajuste (SA) e com ajuste através das covariáveis estimadas pelo método Papadakis $(\mathrm{C} 1, \mathrm{C} 2, \mathrm{C} 3)^{*}$ \{ optimal plot size $\left(\mathrm{X}_{0}\right)$, number of plants, and coefficient of variation among plots of $\mathrm{X}_{0}\left(\mathrm{CV}\left(\mathrm{X}_{0}\right)\right.$, in \%, for the fresh mass of lettuce in experiments under greenhouse, tunnel and in the field, without adjustment (SA) and adjustment by covariates estimated by the method Papadakis (C1, C2, C3)*\}. Santa Maria, UFSM, 2013.

\begin{tabular}{|c|c|c|c|c|c|c|c|c|c|c|}
\hline & $X_{0}$ & $C V_{(x 0)}$ & $X_{0}$ & $C V_{(\mathrm{X} 0)}$ & $X_{0}$ & $C V_{(x 0)}$ & $X_{0}$ & $C V_{(\mathrm{X} 0)}$ & $X_{0}$ & $C V_{(\mathrm{X} 0)}$ \\
\hline & \multicolumn{2}{|c|}{$\begin{array}{c}\text { Estufa } \\
\text { (outono, 2008) }\end{array}$} & \multicolumn{2}{|c|}{$\begin{array}{c}\text { Estufa } \\
\text { (primavera, 2008) }\end{array}$} & \multicolumn{2}{|c|}{$\begin{array}{c}\text { Túnel } 1 \\
\text { (outono, 2008) }\end{array}$} & \multicolumn{2}{|c|}{$\begin{array}{c}\text { Túnel } 1 \\
\text { (primavera, 2008) }\end{array}$} & \multicolumn{2}{|c|}{$\begin{array}{c}\text { Túnel } 2 \\
\text { (outono, 2008) }\end{array}$} \\
\hline$\overline{\mathrm{SA}}$ & 6 & 8,74 & 6 & 12,29 & 7 & 16,37 & 6 & 14,25 & 6 & 12,42 \\
\hline $\mathrm{C} 1$ & 4 & 5,81 & 4 & 8,20 & 5 & 12,00 & 4 & 8,62 & 4 & 7,81 \\
\hline $\mathrm{C} 2$ & 5 & 7,55 & 4 & 10,06 & 6 & 14,04 & 5 & 10,60 & 5 & 10,14 \\
\hline \multirow[t]{2}{*}{$\mathrm{C} 3$} & 5 & 7,55 & 5 & 10,43 & 6 & 14,53 & 5 & 11,13 & 6 & 11,31 \\
\hline & \multicolumn{2}{|c|}{$\begin{array}{c}\text { Túnel } 2 \\
\text { (primavera, 2008) }\end{array}$} & \multicolumn{2}{|c|}{$\begin{array}{c}\text { Estufa } \\
\text { (verão, 2007) }\end{array}$} & \multicolumn{2}{|c|}{$\begin{array}{c}\text { Estufa } \\
\text { (inverno, 2007) }\end{array}$} & \multicolumn{2}{|c|}{$\begin{array}{c}\text { Campo } \\
\text { (verão, 2007) }\end{array}$} & \multicolumn{2}{|c|}{$\begin{array}{c}\text { Campo } \\
\text { (inverno, 2007) }\end{array}$} \\
\hline$\overline{\mathrm{SA}}$ & 5 & 10,80 & 6 & 13,42 & 6 & 13,50 & 8 & 17,39 & 7 & 15.40 \\
\hline $\mathrm{C} 1$ & 4 & 7,91 & 4 & 8,28 & 4 & 10,01 & 5 & 10,97 & 5 & 10,13 \\
\hline $\mathrm{C} 2$ & 4 & 9,90 & 5 & 10,79 & 5 & 12,04 & 6 & 13,98 & 6 & 13,41 \\
\hline $\mathrm{C} 3$ & 4 & 10,04 & 5 & 11,87 & 6 & 12,54 & 7 & 14,90 & 6 & 13.90 \\
\hline
\end{tabular}

$\mathrm{C} 1=$ ajuste com a covariável estimada pela equação $2 ; \mathrm{C} 2=$ ajuste com a covariável estimada pela equação 3 ; $\mathrm{C} 3=$ ajuste com a covariável estimada pela equação 4 .

Papadakis, que possibilita a redução do tamanho da parcela. Além disso, o uso do tamanho de parcela prevendo uso de ANCOVA com covariável estimada pelo

método Papadakis, pode potencializar os benefícios dessa técnica, uma vez que o tamanho de parcela estaria adequado à variabilidade que ocorrerá na área

Tabela 4. Resumo das análises de variância (ANOVA) e covariância considerando a covariável estimada pelo método Papadakis pelas formas 1 (ANCOVA 1), 2 (ANCOVA 2) e 3 (ANCOVA 3), para variável massa fresca de alface a campo s summary of analysis of variance (ANOVA) and covariance considering the covariate estimated by the Papadakis method by the methods 1 (ANCOVA 1), 2 (2 ANCOVA) and 3 (3 ANCOVA) for fresh mass of lettuce in the field\}. Santa Maria, UFSM, 2013.

\begin{tabular}{lccc}
\hline FV & GL & QM & P-valor $^{*}$ \\
\hline \multicolumn{4}{c}{ ANOVA } \\
\hline Bloco & 2 & 20747,087 & 0,000 \\
Tratamento & 15 & 1958,239 & 0,477 \\
Erro & 30 & 1951,840 & \\
\hline \multicolumn{4}{c}{ ANCOVA 1 } \\
\hline Tratamento & 15 & 1958,239 & 0,087 \\
Cov1 & 1 & 26852,435 & 0,000 \\
Erro & 29 & 1093,199 & \\
\hline \multicolumn{4}{c}{ ANCOVA 2 } \\
\hline Tratamento & 15 & 1958,239 \\
Cov2 & 1 & 30191,960 \\
Erro & 29 & 978,043 & 0,053 \\
\hline \multicolumn{4}{c}{ ANCOVA 3 } \\
\hline Tratamento & 15 & 1958,239 \\
Cov3 & 1 & 26699,543 & 0,000 \\
Erro & 29 & 1098,471 & 0,000 \\
\hline
\end{tabular}

$\mathrm{FV}=$ fonte de variação; $\mathrm{GL}=$ graus de liberdade; $\mathrm{QM}=$ quadrado médio; $\mathrm{P}$-valor= nível mínimo de significância associado ao teste $\mathrm{F}$. ( $\mathrm{FV}=$ source of variation; $\mathrm{GL}=$ degrees of freedom; $\mathrm{QM}=$ mean square; $\mathrm{P}$-value $=$ minimum level of significance associated with the test $\mathrm{F}$ ). experimental.

Houve efeito significativo da covariável nas três ANCOVAs realizadas, indicando que é possível o uso dessa técnica com qualquer uma das três formas de cálculo da covariável (Tabela 4). Esse resultado é concordante com os de Cargnelutti Filho et al. (2003), no qual todas as formas de cálculo da covariável geraram uma covariável eficiente para uso na ANCOVA. Apesar disso, a redução do quadrado médio do erro $(\mathrm{QMe})$ proporcionada pela ANCOVA, não foi suficiente para que fosse detectado efeito de tratamento em nenhuma das formas de estimativa da covariável (Tabela 4). Isso ocorreu porque na ANOVA o quadrado médio de tratamento $(\mathrm{QMt})$ apresentou um valor similar ao QMe, levando a um elevado nível mínimo de significância (P-valor) no teste $\mathrm{F}$ para tratamento (Tabela 4). Dessa forma, apesar do P-valor ter reduzido em $88,9 \%$ para a ANCOVA 2, a redução não foi suficiente para a rejeição da hipótese de nulidade em nível de 5\% de probabilidade de erro.

O uso da ANCOVA com a covariável estimada pelo método Papadakis reduziu a estimativa das estatísticas $\mathrm{CV}, \Delta \mathrm{e}$ DMS e aumentou a do $\mathrm{F}_{\text {calculado }}$ nas três formas de estimativa da covariável (Tabela 5), o que ocorreu devido à redução 
Tabela 5. Estatísticas indicadoras de qualidade experimental para a análise de variância (ANOVA) e análise de covariância considerando a covariável estimada pelo método Papadakis pelas formas 1 (ANCOVA 1), 2 (ANCOVA 2) e 3 (ANCOVA 3), com os respectivos aumentos ou reduções em relação à ANOVA, para variável massa fresca de alface a campo \{statistics indicator of experimental quality for the analysis of variance (ANOVA) and analysis of covariance considering the covariate estimated by Papadakis method for methods 1 (ANCOVA 1 ), 2 (ANCOVA 2) and 3 (ANCOVA 3), with respective increases or decreases in the ANOVA for variable fresh mass of lettuce in the field . Santa Maria, UFSM, 2013.

\begin{tabular}{lcccc}
\hline Estatísticas $^{*}$ & ANOVA & ANCOVA 1 & ANCOVA 2 & ANCOVA 3 \\
\hline CV(\%) & 15,225 & $11,394(-25 \%)$ & $10,777(-29 \%)$ & $11,422(-25 \%)$ \\
$\mathrm{F}_{\text {calculado }}$ & 1,003 & $1,791(+79 \%)$ & $2,002(+100 \%)$ & $1,783(+78 \%)$ \\
DMS & 134,423 & $100,982(-25 \%)$ & $95,516(-29 \%)$ & $101,225(-25 \%)$ \\
DMS(\%) & 46,323 & $34,8(-25 \%)$ & $32,916(-29 \%)$ & $34,883(-25 \%)$ \\
\hline
\end{tabular}

${ }^{*}$ Coeficiente de variação residual $(\mathrm{CV} \%)$, valor de $\mathrm{F}_{\text {calculado }}$ para efeito de tratamento, diferença mínima significativa entre dois tratamentos pelo teste de Tukey (DMS) e diferença mínima significativa pelo teste de Tukey em porcentagem da média (DMS\%) \{residual coefficient of variation $(\mathrm{CV} \%), \mathrm{F}_{\text {calculated }}$ for treatment effect, least significant difference between two treatments by the Tukey test (DMS) and least significant differences of Tukey test as a percentage of average $\mathrm{t}(\mathrm{DMS} \%)$ \}.

do QMe (Tabela 4). Esses resultados reforçam a idéia sustentada nesse trabalho de que o uso de ANCOVA com a covariável estimada pelo método Papadakis proporciona melhorias na qualidade de experimentos com a cultura da alface, ficando evidente principalmente pela estimativa do $\mathrm{F}_{\text {calculado }}$ que aumentou em $100 \%$ na ANCOVA 2 (Tabela 5). Esta melhoria na qualidade dos experimentos com alface corrobora com os resultados obtidos para as culturas do milho, soja e feijão (Cargnelutti Filho et al., 2003; Souza et al., 2003; Costa et al., 2005; Storck et al., 2008, 2009, 2010).

O melhor resultado em termos de melhoria dessas estatísticas indicadoras de qualidade experimental foi obtido quando se utilizou a covariável C2 e o pior quando se usou a C3 (Tabela 5). Esse resultado confirma aquele obtido a partir dos experimentos em branco, em que a C3 foi a covariável que conferiu o pior ajuste. No entanto, discorda em relação à melhor forma de cálculo da covariável, uma vez que, nos experimentos em branco, o melhor ajuste foi aquele realizado pela $\mathrm{C} 1$. Apesar dessa discordância, a covariável preferida para uso em ANCOVA em experimentos com alface deve ser a C1, uma vez que essa informação foi obtida através de um banco de dados maior, em que foram contemplados diversos ambientes e épocas de cultivo, e o experimento com tratamentos reflete apenas uma situação específica.

O uso de análise de covariância (ANCOVA) com a covariável estima- da pelo método Papadakis aumenta a qualidade de experimentos com alface e possibilita o uso de menores tamanhos de parcela. Em relação à forma de estimativa da covariável que proporciona a maior eficiência da ANCOVA é aquela que considera uma parcela vizinha de cada lado da parcela de referência no sentido da linha de cultivo (C1). O tamanho de parcela na linha de cultivo, ajustado para uso de ANCOVA com a covariável estimada pelo método Papadakis, considerando a $\mathrm{C} 1$, é de quatro plantas (1,2 m) para estufa e túnel, e de cinco plantas $(1,5 \mathrm{~m})$ para o campo.

\section{AGRADECIMENTOS}

Ao CNPq e a FAPERGS pelo auxílio financeiro para a realização dos experimentos e bolsas de pesquisa e de iniciação científica.

\section{REFERÊNCIAS}

ABCSEM. Projeto para levantamento dos dados socioeconômicos da cadeia produtiva de hortaliças no Brasil 2010/2011. 2011. Campinas, online. Disponível em $<$ http:// www.abcsem.com.br/docs/direitos_resevados. pdf. Acessado em 01 jun. 2015.

BAUMGARTNER D; SAMPAIO SC; SILVA TR; TEO CRPA; VILAS BOAS MA. 2007. Reúso de águas residuárias da piscicultura e da suinocultura na irrigação da cultura da alface. Engenharia Agrícola 27: 152-163.

CARGNELUTTI FILHO A; STORCK L; LÚCIO AD. 2003. Ajustes de quadrado médio do erro em ensaios de competição de cultivares de milho pelo método de Papadakis. Pesquisa Agropecuária Brasileira 38: 467-473.
COSTA JR; BUENO FILHO JSS; RAMALHO MAP. 2005. Análise espacial e de vizinhança no melhoramento genético de plantas. Pesquisa Agropecuária Brasileira 40: 1073-1079.

EMBRAPA. 2006. Centro Nacional de Pesquisa de Solos. Sistema brasileiro de classificação de solos. 2. ed. Rio de Janeiro: Embrapa. 306p.

LORENTZ LH; LÚCIO AD; BOLIGON AA; LOPES SJ; STORCK L. 2005. Variabilidade da produção de frutos de pimentão em estufa plástica. Ciência Rural 35: 316-323.

LÚCIO AD; CARPES RH; STORCK L; LOPES SJ; LORENTZ LH; PALUDO AL. 2008. Variância e média da massa de frutos de abobrinha-italiana em múltiplas colheitas. Horticultura Brasileira 26: 335-341.

LÚCIO AD; HAESBAERT FM; SANTOS D; BENZ V. 2011. Estimativa do tamanho de parcela para experimentos com alface. Horticultura Brasileira 29: 510-515.

MARODIM VS; STORCK L; LOPES SJ; SANTOS OS; SCHIMIDT D. 2000. Delineamento experimental e tamanho de amostra para alface cultivada em hidroponia. Ciência Rural 30: 779-781.

MORENO JA. 1961. Clima no Rio Grande do Sul. Porto Alegre: Secretaria da Agricultura. 41p.

OLIVEIRA NG; DE-POLLI H; ALMEIDA DL; GUERRA JGM. 2006. Plantio direto de alface adubada com cama de aviário sobre coberturas vivas de grama e amendoim forrageiro. Horticultura Brasileira 24: 112-117.

PAPADAKIS JS. 1937. Méthode statistique pour des expériences sur champ. Thessalonike: Institut d'Amélioration des Plantes à Salonique. 30p. (Bullettin, 23).

PARANAIBA PF; FERREIRA DF; MORAIS AR. 2009. Tamanho ótimo de parcelas experimentais: proposição de métodos de estimação. Revista Brasileira de Biometria 27: 255-268

PIMENTEL MS; LANA AMQ; DE-POLLI H. 2009. Rendimentos agronômicos em consórcio de alface e cenoura adubadas com doses crescentes de composto orgânico. Revista Ciência Agronômica 40: 106-112.

RADINB; REISSER JÚNIOR C; MATZENAUER R; BERGAMASCHI H. 2004. Crescimento de 
cultivares de alface conduzidas em estufa e a campo. Horticultura Brasileira 22: 178-181.

SANTOS D; HAESBAERT FM; PUHL OJ; SANTOS JRA; LÚCIO AD. 2010. Suficiência amostral para alface cultivada em diferentes ambientes. Ciência Rural 40: 800-805.

SCHWERTNER DV. 2012. Produção de tomateiro

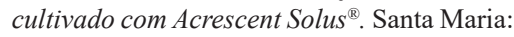
UFSM. 73 p. (Dissertação mestrado).

SOUZA EA; GERALDI IO; RAMALHO MAP;
BERTOLUCCI FLG. 2003. Experimental alternatives for evaluation of progenies and clones in eucalyptus breeding programs. Revista Arvore 27: 427-434.

STORCK L; GARCIA DC; LOPES SJ; ESTEFANEL V. 2006. Experimentação vegetal. 2.ed. Santa Maria: UFSM. 198p.

STORCK L; CARGNELUTTI FILHO A; LÚCIO AD; LOPES SJ. 2009. Método de Papadakis e número de repetições em experimentos de soja. Ciência Rural 39: 977-982.

STORCK L; STECKLING C; ROVERSI T; LOPES SJ. 2008. Utilização do método de Papadakis na melhoria da qualidade experimental de ensaios com soja. Pesquisa Agropecuária Brasileira 43: 581-587.

STORCK L; LOPES SJ; CARGNELUTTI FILHO A; LÚCIO AD; TOEBE M. 2010. Experimental precision in corn trials using the Papadakis method. Ciência e Agrotecnologia 34: 1458-1464. 